\title{
Resgate do conceito de aconselhamento no contexto do atendimento nutricional ${ }^{1}$
}

\author{
Rescuing the concept of counseling within \\ the nutritional attendance context
}

Erika Marafon RODRIGUES²

Fernanda Pardo de Toledo Piza SOARES 3

Maria Cristina Faber BOOG ${ }^{4}$

\section{R E S U M O}

O aconselhamento é um processo genérico de ajuda, cuja estrutura básica independe da área de conhecimento, o qual pode sustentar o atendimento nutricional a grupos e/ou indivíduos, quando então recebe a denominação de aconselhamento dietético. O objetivo do presente trabalho é resgatar o conceito e os fundamentos do aconselhamento, como referência teórica para a atividade de atendimento nutricional que envolve educação e orientação nutricional. A revisão dos fundamentos teóricos que permitiram a construção do modelo básico de aconselhamento, revela a influência e a contribuição relativa de várias correntes da psicologia, além da possibilidade de incorporação do pensamento de educadores identificados com as questões sociais e éticas da educação em saúde. As fases que compõem o processo de aconselhamento incluem: descoberta inicial, exploração em profundidade e preparação para ação. Ao longo do tempo, o foco do trabalho de aconselhamento foi deslocado dos conteúdos técnicos específicos, para a interação de ajuda. Tal deslocamento exigiu o desenvolvimento de competências e habilidades do aconselhador, para compreender e ajudar as pessoas a efetivarem as mudanças necessárias na vida cotidiana e nos construtos pessoais. Por meio desse panorama teórico, conclui-se que o aconselhamento dietético pode prover ao nutricionista um instrumental teórico que Ihe permite aprimorar suas habilidades e competências, com o fim de intervir sobre o comportamento alimentar do cliente, respeitando sua autonomia e valorizando seu potencial como sujeito histórico.

Termos de indexação: aconselhamento dietético, educação em saúde, educação nutricional, nutricionista.

\footnotetext{
1 Artigo elaborado a partir da dissertação em Enfermagem de E.M. RODRIGUES. "Problematização do comportamento alimentar como estratégia de educação nutricional: uma experiência com adolescentes obesos". Departamento de Enfermagem, Faculdade de Ciências Médicas, Universidade Estadual de Campinas, 2003.

2 Nutricionista, Mestre em Enfermagem. Consultório particular. Piracicaba, SP, Brasil.

3 Nutricionista, Universidade Presidente Antonio Carlos. Juiz de Fora, MG, Brasil.

4 Departamento de Enfermagem, Faculdade de Ciências Médicas, Universidade Estadual de Campinas. Caixa Postal 6111, 13083-970, Campinas, SP, Brasil. Correspondência para/Correspondence to: M.C. FABER BOOG. E-mail: <crisboog@fmc.unicamp.br>.
} 


\section{A B S T R A C T}

Counseling is a general process of help, whose basic structure is not dependent on the area of knowledge; when it supports the nutritional attendance to groups and/or individuals, it is named dietary counseling. The goal of this work is to rescue the concepts and fundamentals of counseling as a theoretical reference to the activity of nutritional attendance, which involves education and nutritional orientation. The review of the theoretical requirements, which have allowed the design of the basic counseling model, reveals the influence and the relative contribution of several lines of psychology. In addition, there is the possibility of incorporating the rationale of educators, related to the social and ethic education issues in health. The stages comprising the counseling process include: initial disclosure, in-depth exploration and preparation for action. Along the years, the focus of counseling work has shifted, from the specific technical contents to the interaction of help, . such interaction has demanded the development of the counselor's qualifications and ability, in order to understand people and to help them carry out those changes found, necessary in the everyday life and people constructs. Reviewing the theoretical scenario, one concludes that dietary counseling can supply nutritionists with a theoretical tool to improve their skills and qualifications, in order to interfere with the client's feeding behavior, taking their autonomy into account and recognizing their potential as historic subjects.

Indexing terms: dietary counseling, health education, nutrition education, nutritionist.

\section{N T R O D U Ç Ã O}

A demanda por orientação alimentar tem crescido significativamente, face ao diagnóstico precoce das doenças crônicas e ao reconhecimento da influência da alimentação sobre elas. Também, a consideração do sobrepeso e da obesidade como fatores de risco para doenças cardiovasculares, hipertensão, diabetes mellitus, osteoartrite, osteoporose, câncer de mama, de endométrio e de cólon ${ }^{1,2}$, leva à procura crescente de atendimento nutricional.

Não há ainda registros sobre o incremento e a dimensão atual dessa demanda, mas observase que ela tem aumentado tanto na rede básica de saúde, quanto em clínicas e consultórios. O aumento da demanda por "orientação nutricional", "orientação alimentar", "consulta de nutrição" ou "atendimento nutricional", justifica a retomada do estudo do aconselhamento dietético, conceituado, em 1969, pela Associação Americana de Dietética, como a "orientação profissional individualizada para ajudar uma pessoa a ajustar seu consumo diário de alimentos, a fim de atender às necessidades de saúde" ${ }^{3}$.

No presente trabalho, as autoras propõem abordar o tema quanto aos seguintes aspectos: fundamentação teórica do aconselhamento, histórico do aconselhamento dietético, etapas do processo e comunicação não verbal. Pressupõe-se que a questão a ser tratada no aconselhamento dietético não é a mera "adesão à dieta": busca-se, em conjunto com o cliente, encontrar estratégias para solucionar problemas relativos ao comportamento alimentar, problemas estes, que englobam práticas alimentares inadequadas, hipóteses relativas às práticas alimentares inadequadas levantadas a partir da história alimentar, dos dados clínicos, bioquímicos ou antropométricos do cliente, bem como qualquer questão de caráter subjetivo que possa gerar nele a dúvida, a ansiedade e a insegurança quanto aos efeitos dos alimentos ou nutrientes sobre o organismo, aventados ou efetivamente percebidos como sinais ou sintomas.

\section{F U N D A M E N T A ÇÃ O TE Ó RICA DO ACONSELHAMENTO}

O aconselhamento dietético tem sua fundamentação teórica em um modelo genérico de aconselhamento o qual, segundo Patterson \& Eisenberg ${ }^{4}$, foi estruturado a partir das linhas 
teóricas da psicologia, sucintamente descritas a seguir:

Aconselhamento Centrado no Cliente: esta abordagem propicia ao cliente o desenvolvimento de valores positivos, contribuindo para um comportamento integrado. A visão que a pessoa tem de si mesma, no contexto do meio, determina seu comportamento. As etapas neste processo devem colaborar para a auto-descoberta. As características centrais do conselheiro são empatia e congruência. O enfoque centrado no cliente prioriza os fatos e condições atuais. Situações anteriores, mesmo as estreitamente ligadas ao sujeito, como as relacionadas ao vínculo mãe-filho, ou outras situações problemáticas vividas anteriormente à atual, não são trabalhadas, mas sim aquelas que o indivíduo experimenta em seu tempo real.

Aconselhamento Gestáltico: a ênfase desta linha psicológica é sobre o organismo como um todo, buscando o equilíbrio, que é constantemente ameaçado por circunstâncias externas e conflitos interiores. Esta proposição engloba tomada de atitudes que colocam a pessoa em uma re-configuração, compreendendo polaridades dentro do seu meio. Tais polaridades se evidenciam por meio de atitudes, posturas, comportamentos, reflexões, ações sobre saúde/doença, relações indivíduo-sociedade e relações interpessoais. Do ponto de vista da nutrição, é necessário compreender os diversos significados que o indivíduo atribui às limitações da possibilidade do controle dietético para a resolução dos problemas relativos ao comportamento alimentar.

Aconselhamento Psicanalítico: segundo esta linha teórica as pessoas são movidas por um desejo instintivo de satisfazer o prazer pessoal. A libido é a fonte de energia impulsionadora dos comportamentos que satisfazem o prazer. É fundamentado em Freud, que concebeu a estrutura da personalidade dividida em id, ego e supergo. O aconselhamento focaliza o ego, parte da personalidade em contato com a realidade exterior, sede do pensamento racional, que deve também influenciar o comportamento alimentar.
Aconselhamento Racional-Emotivo: o desenvolvimento desta linha teórica pretende que o aconselhamento ajude a pessoa a eliminar os pensamentos auto-destrutivos, promovendo visões de si mesma mais tolerantes e racionais. Como as pessoas possuem poder e tendência para interagir em ambientes de irracionalidade e racionalidade, a emoção negativa decorreria de pensamentos irracionais. O conselheiro, portanto, deve mostrar-se muito ativo neste tipo de relacionamento. O esquema A-B-C pode explicar como o indivíduo identifica ou classifica as conseqüências de determinados fatos, sendo $A$ : o fato, B: a crença ou o significado expresso por ele, e C: a conseqüência. Um exemplo desse pensamento é o da pessoa portadora de diabetes $(A)$, que se torna profundamente infeliz (C) por ter de fazer algumas restrições no seu comportamento alimentar (B). Esta linha pretende ajudar o cliente a reconsiderar os pressupostos sobre seus desejos.

Aconselhamento Baseado em Traços e Fatores: esta foi a linha mais empregada para auxiliar pessoas a fazerem escolhas educacionais e vocacionais. O conselheiro desempenha um papel privilegiado no processo, utilizando suas habilidades especializadas para ajudar o cliente a avaliar fatores implicados na solução de problemas e na tomada de decisão; nesta linha de aconselhamento, as perturbações emocionais, geralmente não constituem um fator significativo. No campo da nutrição, caberiam como exemplos de clientes e situações para os quais este aconselhamento é mais adequado, tanto o atleta profissional que necessita orientação nutricional altamente especializada, como o indivíduo adulto, sadio, compelido a viver só, que busca orientação nutricional a fim de organizar da forma mais prática e racional o atendimento à sua demanda alimentar.

Aconselhamento Behaviorista: tem como objetivo principal a mudança do comportamento inadequado do cliente; apenas o que é mensurável é considerado evidência de aconselhamento bem sucedido. Enfatiza-se a definição e o alcance do 
objetivo. Quando o cliente adquire novas práticas alimentares, ainda que condicionado por meio de reforço positivo (prêmio, elogio) ou negativo (punição, censura), pode-se entender que o processo ocorreu.

Segundo Holli \& Calabrese ${ }^{5}$, autores que publicaram um livro sobre Aconselhamento Dietético há quase duas décadas, a contribuição dessas várias abordagens permitiu estabelecer algumas diretrizes básicas, norteadoras da natureza do trabalho de aconselhamento dietético, citadas a seguir: a consideração de elementos significativos da realidade pessoal; a responsabilidade que o cliente deve ter em relação à sua própria vida; a não dominação do conselheiro sobre o cliente; a explicitação da natureza das dificuldades com base nos diagnósticos; a revelação do ego ao conselheiro para possível interpretação das experiências do cliente; maior percepção do cliente sobre si mesmo; apoio direto às mudanças; mudanças num período curto de tempo; diagnóstico simplificado; vínculo entre o profissional e o cliente.

Aconselhar, no campo da alimentação, torna-se possível e imperativo diante de duas realidades: primeira, a necessidade de recusar modelos dogmáticos, padronizados, lacônicos, pautados, sobretudo, em restrições e normas que pressupõem um comportamento heterônomo do cliente; segunda, a perspectiva de poder inserir as ações educativas de nutrição em um processo comprometido com a compreensão da condição humana, na acepção de Morin ${ }^{6}$, considerando os valores culturais e os indivíduos como sujeitos históricos, além da possibilidade de uma relação horizontal entre educador-educando (profissional-cliente), concepções estas, encontradas também na pedagogia da autonomia de Paulo Freire?. Segundo Morin, "o ser humano é, a um só tempo, físico, biológico, psíquico, cultural, social, histórico" e, desse modo, "a condição humana deveria ser o objeto essencial de todo o ensino" e também de toda prática profissional, inclusive no campo da nutrição. De Paulo Freire, ressalta-se a ênfase nos aspectos inerentes à realidade de vida do educando, no diálogo entre educador e educando, na problematização do comportamento alimentar e na submissão da ciência e da técnica às necessidades, tais quais sentidas e percebidas pelos homens na concretude da sua existência.

Considera-se que a ajuda para resolver problemas alimentares insere-se numa busca de âmbito maior por qualidade de vida. Esta ocorre em determinado momento crítico, no qual surge um problema, que, por sua vez, também é fruto da história de vida. O aconselhamento dietético pode, se convenientemente conduzido, constituir uma oportunidade para a busca ativa de uma vida mais prazerosa e mais plena. Vale ressaltar que o acesso ao aconselhamento nutricional, freqüentemente, se dá em decorrência de uma doença já instalada; entretanto, a educação em saúde voltada aos aspectos relativos à alimentação, deveria fazer parte das estratégias de promoção à saúde ${ }^{8}$. Portanto, a metodologia aqui proposta identifica-se com a visão da educação em saúde como capacitação dos indivíduos e da comunidade para a busca ativa da saúde e de condições para uma existência digna.

\section{HISTÓRICO DO A CONSELHAMENTO D I E TÉ T I C O}

O aconselhamento é uma estratégia educativa que precisa ser resgatada, a fim de redimensionar a atuação do nutricionista junto ao cliente que necessita mudar seu comportamento alimentar. Tal estratégia educativa favorecerá uma vida mais saudável e prazerosa aos indivíduos portadores de doenças que exigem mudança no padrão de alimentação. Desde o ano de 1975, quando a Associação Americana de Dietética posicionou-se a respeito, vários autores e pesquisadores se detiveram sobre o tema e, com base nas contribuições das ciências humanas, foram produzidos trabalhos que possibilitaram ampliar a compreensão da natureza dessa intervenção e explicitar algumas estratégias a serem adotadas para ajudar pessoas que 
necessitam fazer modificações no seu padrão alimentar e estilo de vida.

Orkow \& Ross ${ }^{9}$ relataram uma intervenção desenvolvida por um nutricionista e um assistente social, com grupo de obesos, em que se agregaram educação nutricional e técnicas de aconselhamento, utilizando incentivos psicológicos, instruções dietéticas e exercícios. Os autores fundamentaram o processo em uma abordagem comportamentalista, enfatizando a mudança de hábitos, estes entendidos, pelos autores citados, como práticas alimentares.

Evans \& Hall ${ }^{10}$, trabalhando com psicologia social, procuraram diferenciar a orientação nutricional fundamentada no "modelo médico" (compreendido como aquele em que o cliente segue as orientações do nutricionista que o atende), daquela fundamentada na aliança terapêutica, que garante a autonomia do paciente, pois, desde o início do processo, ele é incentivado a realizar suas próprias escolhas. Nessa abordagem, é relevante a sensibilidade do profissional para a escuta, a sua disposição para o diálogo, a predisposição à formação de vínculo. O "modelo médico" se identifica com a abordagem tradicional de educação fundamentada na transmissão oral de conhecimento, entre alguém que detém o conhecimento e o transfere, e aquele que não o tem e o recebe ${ }^{11}$.

Danish et al. ${ }^{12}$ publicaram os resultados de uma pesquisa, na qual foram avaliados os procedimentos de graduandos de Nutrição durante sessões de Aconselhamento Dietético. Os autores verificaram a predominância de questões fechadas e o pouco uso de expressão não-verbal. Considerando esses resultados como indicativos do emprego inadequado das técnicas, devido à falta de prática dos estudantes, sugeriram a possibilidade de desenvolver nos estudantes a habilidade para o aconselhamento, mediante o ensino e a prática das técnicas adequadas. A mesma metodologia foi aplicada na Universidade da Pensilvânia13, apresentando resultados que mostraram ser a ansiedade, um dos grandes problemas sentidos pelos alunos de nutrição no momento do aconselhamento. Os 25 participantes da pesquisa indicaram sofrer desconforto ao assumir o papel de conselheiro. Os autores sugerem o desenvolvimento de intervenções pedagógicas, com o objetivo de reduzir essa ansiedade excessiva.

Vickery \& Hodges ${ }^{14}$ criaram um esquema bastante elucidativo para descrever o processo de aconselhamento; porém, referiram-se à educação apenas como uma etapa do aconselhamento - a etapa em que o nutricionista informa o cliente e oferece reforço positivo para as mudanças por ele efetuadas. Para as autoras do presente estudo, a educação é mais ampla do que o aconselhamento e este constitui uma estratégia para um determinado tipo de intervenção educativa em nutrição - quando uma pessoa busca ajuda para modificar seu comportamento alimentar.

Outra contribuição interessante veio de Isselmann et al. ${ }^{15}$. Estes, após avaliarem os resultados de um treinamento, em que os participantes discutiram técnicas de aconselhamento, recomendaram aos profissionais que, ao implementarem estes serviços, se assegurassem de que o mesmo ocorreria em espaço e ambiente apropriados, garantindo privacidade e o mínimo de conforto.

Hauchecorne et al. ${ }^{16}$ elaboraram um questionário, com perguntas abertas e fechadas, para investigar a percepção dos clientes sobre o aconselhamento nutricional. Esse instrumento media as percepções dos entrevistados sobre a efetividade e o valor do aconselhamento nutricional, sobre a ocorrência de mudanças das práticas alimentares depois do aconselhamento, e se esse surtia algum efeito não intencional no tratamento. De acordo com os autores, o aconselhamento nutricional era eficaz e a intervenção do nutricionista foi significativa, contribuindo para o bem estar físico e psicossocial dos clientes. Detectaram, ainda, a necessidade de estudos que possibilitassem identificar barreiras e fatores facilitadores para mudanças no comportamento alimentar, e ressaltaram a importância desse tipo de estudo para justificar a manutenção 
dos serviços de aconselhamento e a obtenção de recursos para os mesmos.

Schiller et al. ${ }^{17}$, da Universidade Estadual de Ohio, utilizaram o instrumento elaborado por Hauchecorne et al., junto a 400 pacientes adultos, sendo 274 internos e 126 ambulatoriais. Oitenta e oito por cento dos pacientes reportaram que as orientações nutricionais dadas pelos nutricionistas atenderam às suas necessidades específicas, 83\% referiram que sabiam o que comer, $79 \%$ referiram compreensão boa ou moderada das orientações, $62 \%$ implementaram efetivamente mudanças em sua alimentação, 57\% referiram melhora no estado emocional, $37 \%$ no estado físico e $43 \%$ apresentaram melhora global nas condições de saúde dentro do período de estudo, que foi de duas a oito semanas após o aconselhamento.

Outros autores também demonstraram a eficácia no controle de doenças crônicas, após o período de intervenção nutricional fundamentada nos preceitos do aconselhamento dietético ${ }^{9,18,19}$. Um ponto comum entre eles é a referência ao aconselhamento dietético como um processo de ajuda, cuja proposta central é o encorajamento do cliente para relatar seus problemas.

Recentemente, surgiu na literatura o emprego de novas tecnologias de comunicação para aconselhamento dietético por intermédio de e-mail, telefone e sites na Internet ${ }^{20}$. Conhecendo todos os preceitos propostos para o aconselhamento, lembrando, principalmente, da ênfase dada ao vínculo que deverá existir entre profissional e cliente por meio do contato pessoal - inclusive da linguagem não verbal - causa estranheza o emprego de meios tecnológicos para esta atividade que tem como pressupostos a criação de vínculo e o diálogo. Cabe alertar para a necessidade de encontros que contemplem a formação de vínculo entre profissional e cliente, que precedam o atendimento instrumentalizado pela tecnologia de comunicação.

Destaca-se ainda, a distinção que Boog ${ }^{21}$ faz entre orientação e educação nutricional: a primeira expressão, orientação, significa o fazer imediato, as instruções propriamente ditas, dietas com objetivos específicos e com certo rigor para horários e técnicas. Entretanto, a educação nutricional volta-se para a formação de valores, para o prazer, a responsabilidade, a atitude crítica, assim como para o lúdico e a liberdade.

O conceito de aconselhamento dietético chegou a ser divulgado no Brasil, por intermédio de Cavalcanti22, Motta \& Boog ${ }^{23}$ e Martins ${ }^{24}$, porém, sem grande repercussão, uma vez que essa terminologia é pouco utilizada e desconhecida pelos profissionais da área.

Observa-se, até mesmo, uma resistência às discussões sobre os pressupostos teóricos das ações educativas. É interessante salientar que o fato não ocorre só aqui. Achterberg \& Clark ${ }^{25}$ avaliaram 346 pesquisas sobre Educação Nutricional, publicadas no Journal of Nutrition Education, entre 1980 e 1990, verificando que $75 \%$ delas não explicitavam a teoria que as sustentava. Essa resistência precisa ser enfrentada, pois a ampliação da compreensão dos processos de trabalho contribui de forma significativa ao aprimoramento das ações. Ao fazer educação nutricional não se está apenas lidando com nutrientes, mas com todo o universo de interações e significados que compõe o fenômeno do comportamento alimentar, conforme conceituado por Garcia (p.12)26:

procedimentos relacionados às práticas alimentares de grupos humanos (o que se come, quanto, como, quando, onde e com quem se come; a seleção de alimentos e os aspectos referentes ao preparo da comida) associados a atributos sócio-culturais, ou seja, aos aspectos subjetivos individuais e coletivos relacionados ao comer e à comida (alimentos e preparações apropriadas para situações diversas, escolhas alimentares, combinação de alimentos, comida desejada e apreciada, valores atribuídos a alimentos e preparações e aquilo que pensamos que comemos ou que gostaríamos de ter comido). 
Optou-se pelo uso desse conceito após cuidadosa análise do significado da palavra comportamento, na linguagem comum e no domínio da psicologia. Em dicionário consultado, de língua portuguesa, ele é referido como "maneira de se comportar, procedimento, conduta", englobando o "conjunto de atitudes e reações do indivíduo em face do meio social". Para a psicologia, significa "o conjunto das reações que se podem observar num indivíduo estando este em seu ambiente, e em dadas circunstâncias"27. As ciências humanas apropriaram-se do termo, atribuindo a ele conotações mais específicas. O dicionário de sociologia designa comportamento como "a mudança, o movimento ou a reação de qualquer entidade ou sistema em relação a seu ambiente ou situação" 28 . Considerando que comportamento, na língua portuguesa, ou behaviorismo, na língua inglesa, são palavras anteriores às escolas psicológicas, e que, portanto, elas não constituem prerrogativas exclusivas de quaisquer teorias e, ainda, a inexistência de outra palavra mais abrangente que explicite com maior clareza o fenômeno a ser estudado, optou-se por tomá-la como referência para o objeto do aconselhamento dietético.

Tonial ${ }^{29}$ observa que os manuais de "dietas universais" são padronizados e suas receitas, prescritas pelos profissionais de saúde, tanto nas Unidades da Europa, como nos Postos de Saúde de qualquer longínqua e interiorana região do Brasil. Ou seja, os modelos técnicos que pretendem tratar o indivíduo enquanto doença, são similares em qualquer região do mundo; no entanto, o molde no qual está inserida a orientação, pode ser diferenciado por meio de um novo olhar que trate o indivíduo enquanto sujeito de sua história, tendo o aconselhamento dietético como referência deste tipo de atendimento. A prática de diferentes profissionais não parece diferir muito, conforme demonstram Amorim et al. ${ }^{30}$. Em seu trabalho, realizado com nutricionistas e pediatras, os autores expõem a forma tradicional em que esses profissionais atendem. Indicam a necessidade de virem esses profissionais a conhecer processos que envolvam uma constituição mais humana da prática, eliminando, desta forma, conceitos e comportamentos reducionistas, os quais fragmentam o ser humano (o cliente) em corpo e mente.

Com base na fundamentação teórica e nos trabalhos já publicados a respeito, as autoras apresentam a seguir uma proposta de abordagem para o aconselhamento dietético.

\section{ETAPAS DO PROCESSO}

Descoberta Inicial: esta fase influencia sobremaneira a condução do relacionamento entre cliente e nutricionista, pois caracteriza a formação do vínculo. Empatia, autenticidade, considerações positivas, incondicionalidade e concreção, são atributos importantes na qualidade do encontro. O nutricionista deve estar preparado para captar o estado emocional do cliente (ansiedade, nervosismo, insatisfação) declarado verbalmente ou por meio de gestos, posturas, movimentos do corpo, expressões faciais, qualidade da voz e o silêncio. O profissional deve, antes de tudo, saber ouvir e saber aceitar, criando um ambiente favorável para a construção de estratégias que favoreçam o desenvolvimento de ações pelo cliente.

Exploração em Profundidade: esta etapa deve, basicamente, servir para encorajar o cliente para a formação de insight, favorecendo uma condição de discernimento e tentativa de discussão sobre os problemas. O nutricionista precisa estar preparado para enfrentar os obstáculos que poderão bloquear a articulação das idéias pelo cliente, visto que este, muitas vezes, poderá transferir-se para um campo imaginário no qual moldará no conselheiro uma figura de autoridade - revelando, desta maneira, os modelos frustrados de atendimento, pré-existentes em si. Para que tal situação não perdure, é imprescindível que o profissional, durante o atendimento, seja sensível para perceber a necessidade que o cliente tem de ser encorajado, a fim de poder desvelar e 
ampliar os temas relacionados ao problema. Para uma ajuda efetiva, o nutricionista precisa compartilhar as experiências apontadas pelo cliente, expandindo os conceitos revelados. Várias técnicas podem ser utilizadas, no intuito de facilitar a abordagem ao tema: questões fechadas (que normalmente são limitadas e não permitem exploração do tema), questões abertas (que estimulam respostas mais longas e reflexivas), uso de diretivas (incentivos utilizados pelo nutricionista quando o cliente não formula a idéia), uso de estímulos (habilidades não verbais que indicam atenção e compreensão) e escuta efetiva. Estas técnicas têm como objetivo principal direcionar a interação de forma a problematizar o cotidiano alimentar.

Preparação para a ação: este estágio pode causar certa ansiedade no cliente que não está acostumado a aventar estratégias próprias para solucionar seus problemas. Algumas barreiras devem ser rompidas, a fim de que o cliente tome decisões quanto a como realizar os objetivos e ações. A existência de um feedback autêntico, por meio de elogios, pode ser um elemento constitutivo da boa relação cliente-conselheiro. Como resultado final do aconselhamento, o cliente fará planos para corrigir o problema, lembrando que as duas primeiras fases foram interrogativas para ele. Esta etapa de formulação da solução pode exigir tempo, disciplina e paciência. É importante ressaltar a necessidade de uma avaliação conjunta, pelo nutricionista e pelo cliente, das estratégias selecionadas para enfrentar os problemas, dos resultados obtidos e das mudanças conjunturais.

Alcançar essas três fases indica que o cliente, possivelmente, encontrou estratégias para solucionar seu problema; no entanto, isso só será constatado a partir de encontros freqüentes com o nutricionista, nos quais este avaliará as mudanças no pensar, no sentir e no agir do cliente, podendo ainda haver necessidade de retomarem as etapas anteriores na discussão de questões ainda não problematizadas.

\section{O M U N I C A ÇÃ O N Ã O VER B A L}

O aconselhador deverá estar em sintonia com o cliente, sempre observando sua fala e suas atitudes. A postura do corpo é uma linguagem silenciosa de comunicação não-verbal, e por meio de expressões faciais, gestos, movimentos corporais, tom de voz, velocidade da fala, afinação de voz, entre outros indícios, pode-se identificar o posicionamento do cliente, subjacente ao problema ${ }^{31,32}$.

O silêncio é um dos fatores, citados por, Hackney \& Nye ${ }^{32}$, que exigem atenção, pois tanto pode significar que o cliente está examinando a si próprio, como pode indicar que ele queira evitar o assunto. Conforme Weil \& Tompakow ${ }^{31}$, "pela linguagem do corpo, você diz muitas coisas aos outros. E eles têm muitas coisas a dizer para você. Também nosso corpo é, antes de tudo, um centro de informações para nós mesmos. É uma linguagem que não mente".

Hackney \& Nye $^{32}$ pontuam que, os aconselhadores e os clientes, no processo de entrevista, transmitem e recebem mensagens verbais e não verbais continuamente. O aconselhador deve demonstrar que presta atenção (é uma postura que afirma comprometimento com o cliente, conduzindo-o a sentir segurança), manifestando-se em expressões faciais (olhar direto, meneios de cabeça, jogo dos músculos faciais para produzir rugas, olhares interrogativos, indiferença, entre outras expressões), mas tendo cuidado no comportamento verbal (a fala do aconselhador tem efeito imediato sobre o cliente; portanto, aquele deve evitar o uso de expressões como "hum-hum", "eu entendo", pois estes estímulos verbais podem produzir um bloqueio na comunicação).

\section{O N CLUSÃO}

As autoras recomendam a adoção do termo "aconselhamento dietético" para a designação da atividade de consulta de nutrição, por estar fundamentado teoricamente em 
bibliografia, tanto da área de ciências humanas, quanto da área específica de nutrição. Esta modalidade de intervenção de educação nutricional, requer encontros freqüentes para a execução efetiva das etapas de todo o processo. Exige ainda a garantia da privacidade do cliente, ou do grupo, como atributo ético no atendimento. Ressalta-se também que as habilidades e competências do aconselhador devem constituirse de uma junção de conhecimentos específicos de nutrição, de educação nutricional, e de conhecimentos advindos das ciências humanas, especialmente da antropologia da alimentação. O preparo dos nutricionistas para essa modalidade de intervenção demanda estágios práticos, supervisionados por profissionais dessa área, que os auxiliem a perceber a intersubjetividade no relacionamento com o cliente, e a explorar as possibilidades e compreender as limitações da comunicação verbal e não verbal, de forma a instrumentalizá-los para lidar com o inesperado e, sobretudo, com as próprias inseguranças.

Sintetizando as considerações aqui expostas, conceitua-se o aconselhamento dietético como uma abordagem de educação nutricional, efetuada por meio do diálogo entre o cliente portador de uma história de vida - que procura ajuda para solucionar problemas de alimentação - e o nutricionista, preparado para analisar o problema alimentar no contexto biopsicossociocultural da pessoa, que a auxiliará a explicitar os conflitos que permeiam o problema, a fim de buscar soluções que permitam integrar as experiências de criação de estratégias para o enfrentamento dos problemas alimentares na vida cotidiana, buscando um estado de harmonia compatível com a saúde.

\section{REFERÊ N CIAS}

1. World Health Organization. Study group on diet, nutrition and prevention of non-communicable diseases. Geneve; 1989. (Technical Report Series, 797).
2. Meisler JG, Jeor SS. Forward. Am J Clin Nutr. 1996; 63(3 Suppl):409-11.

3. Ling L, Sprag D, Stein P, Myers ML Guidelines for diet counseling. J Am Diet Assoc. 1975; 66(6): 571-5.

4. Patterson LE, Eisenberg S. O Processo de aconselhamento. São Paulo: Martins Fontes; 1988.

5. Holli BB, Calabrese RJ. Communication and education skills: the dietitian's guide. Philadelphia: Lea \& Febiger; 1986.

6. Morin E. Os sete saberes necessários à educação do futuro. 3.ed. São Paulo: Cortez; 2001.

7. Freire P. Pedagogia da autonomia: saberes necessários à prática educativa. São Paulo: Editora Paz e Terra; 1998.

8. Buss PM. Promoção da saúde e qualidade de vida. Ciênc \& Saúde Coletiva. 2000; 5(1):163-77.

9. Orkow BM, Ross JL. Weight reduction through nutrition education and personal counseling. J Nutr Educ. 1975; 7(2):65-7.

10. Evans Rl, Hall Y. Social-psychological perspective in motivating changes in eating behavior. J Am Diet Assoc. 1978; 72(4):378-83.

11. Mizukami MGN. Ensino: as abordagens do processo. São Paulo: EPU; 1986.

12. Danish SJ, Ginsberg MR, Terrel A, Hammond MI, Adams SO. The anatomy of a dietetic counseling interview. J Am Diet Assoc. 1979; 75(12):626-30.

13. Greene GW, Yesenosky J. Preparing nutrition counselors: perceptions of dietetic students in counseling practicum. J Am Diet Assoc. 1990; 90(2):274-6.

14. Vickery C, Hodges PAM. Counseling strategies for dietary management: expanded possibilities for effecting behavior change. J Am Diet Assoc. 1986; 86(7):924-8.

15. Isselmann MC, Deubner LS, Hartman M. A nutrition counseling workshop: integrating counseling psychology into nutrition practice. J Am Diet Assoc. 1993; 93(3):324-6.

16. Hauchecorne CM, Barr SI, Sork TJ. Evaluation of nutrition counseling in clinical settings: Do we 
make a difference? J Am Diet Assoc. 1994; 94(4):437-40.

17. Schiller MR, Miller M, Moore C, Davis E, Dunn A, Mulligan $\mathrm{K}$, et al. Patients report positive nutrition counseling outcomes. J Am Diet Assoc. 1998; 98(9):977-82.

18. Bakx JC, Stafleu A, van Staveren WA, van den Hoogen HJM, van Weel C. Long-term effect of nutritional counseling: a study in family medicine. Am J Clin Nutr. 1997; 65 Suppl:1946S-50S.

19. Gilboy MB. Multiple factors affect dietitians' counseling practices. J Am Diet Assoc. 1994; 94(11):1278-83.

20. Palumbo C. Using new technology for nutrition counseling. J Am Diet Assoc. 1999; 99(11): 1363-4.

21. Boog MCF. Educação Nutricional: passado, presente e futuro. Rev Nutr. 1997; 10(1):5-19.

22. Cavalcanti MLF. A entrevista alimentar como método educativo na orientação dietoterápica de pacientes externos. Rev Paul Hosp. 1976; 24: 516-25.

23. Motta DG, Boog MCF. Educação nutricional. 2.ed. São Paulo: Ibrasa; 1988.

24. Martins C. Aconselhamento dietético. In: Riella MC, Martins C. Nutrição e o rim. Rio de Janeiro: Guanabara-Koogan; 2001.
25. Achterberg C, Clark KL. A retrospective examination of theory use in nutrition education. J Nutr Educ. 1992; 24(5):227-33.

26. Garcia RWD. A comida, a dieta, o gosto: mudanças na cultura alimentar urbana [Tese]. São Paulo: Instituto de Psicologia, Universidade de São Paulo; 1999.

27. Comportamento. In: Dicionário Aurélio Eletrônico - Século XXI. Versão 3.0 [CD-Rom]. Rio de Janeiro: Nova Fronteira; 1999.

28. Comportamento. In: Dicionário de Ciências Sociais. Rio de Janeiro: Editora da Fundação Getúlio Vargas; 1986.

29. Tonial SR. Desnutrição e obesidade: faces contraditórias na miséria e na abundância. Recife: IMIP; 2001.

30. Amorim STSP, Moreira H, Carraro TE. A formação de pediatras e nutricionistas: a dimensão humana. Rev Nutr. 2001; 14(2):111-8.

31. Weil P, Tompakow R. O corpo fala. Petrópolis: Vozes; 1996.

32. Hackney H, Nye S. Aconselhamento: estratégias e objetivos. São Paulo: EPU; 1977.

Recebido para publicação em 1 de julho de 2003 e aceito em 3 de fevereiro de 2004. 